\title{
PERCEPÇÕES DOS PAIS SOBRE OS SINTOMAS DO TDAH EM CRIANÇAS E ADOLESCENTES DIAGNOSTICADOS COM TEA
}

\section{PARENTS PERCEPTIONS ON ADHD SYMPTOMS IN CHILDREN AND ADOLESCENTS DIAGNOSED WITH ASD}

\author{
Elma Dayana Moura dos Santos ${ }^{1}$, \\ Ana Flávia Lima Teles da Hora ${ }^{2}$
}

\begin{abstract}
RESUMO: O transtorno do Déficit de Atenção e Hiperatividade (TDAH) tem sido alvo de inúmeros questionamentos na contemporaneidade, visto que este acarreta um comprometimento em diversos aspectos da vida do sujeito. A presente pesquisa caracteriza-se como um estudo preliminar, de cunho exploratório e caráter quantitativo, tendo como objetivo identificar a percepção dos pais no que tange aos sintomas do TDAH em crianças e adolescentes com diagnóstico de TEA (Transtorno do Espectro Autista). Esta foi aplicada na clínica escola Ana Lúcia Chaves Fecury, da Universidade CEUMA, localizada em São Luís - MA. O critério de escolha dos participantes consistiu em pais que possuem filhos com idade de 6 a 12 anos com o diagnóstico clínico de TEA. Na coleta de dados, foram utilizados os seguintes instrumentos: a Escala CBCL/6-18 (Child Behavior Checklist) e o questionário SNAP-IV (Swanson, Nolan e Pelham - versão IV). No processo de análise de dados foi utilizado o software SPSS (Statistical Package for Social Sciense), versão 22.0. Os resultados encontrados sinalizam para a existência de sintomas de desatenção em crianças e adolescentes autistas de acordo com a percepção dos pais. A pesquisa contribuiu para a ampliação dos estudos concernentes à temática TDAH, assim como para apontar possíveis correlações existentes entre o TEA e o TDAH. Sugere-se, para futuras pesquisas, a realização de estudos referentes à prevalência do TDAH como transtorno comórbido do TEA, bem como a ampliação da amostra clínica.
\end{abstract}

PALAVRAS-CHAVE: Autismo; Pais; CBCL.

ABSTRACT: The Attention Deficit Hyperactivity Disorder (ADHD) has been the subject of numerous questions in the contemporary world, since this entails a compromise in several aspects of the subject's life. The present research is characterized as a preliminary exploratory and quantitative study, aiming to identify the parents' perception regarding the symptoms of ADHD in children and adolescents diagnosed with ASD (Autistic Spectrum Disorder). The same was applied at the Ana Lúcia Chaves Fecury clinical school, of the Ceuma University. The choice of the participants consisted of parents who have children age 6 to 12 years with clinical diagnosis of ASD. The following instruments were used in the data collection: The CBCL / 6-18 Scale (Child Behavior Checklist) and the SNAP-IV questionnaire (Swanson, Nolan and

\footnotetext{
${ }^{1}$ Graduanda do Curso de Psicologia, cursando o $10^{\circ}$ período na Universidade Ceuma. Email: elmadayanna.moura@outlook.com

${ }^{2}$ Psicóloga. Doutora e Mestre em Teoria e Pesquisa do Comportamento pela Universidade Federal do Pará (UFPA). Professora e pesquisadora na Universidade Ceuma e no Núcleo de Tecnologia para Educação (UEMANET) da Universidade Estadual do Maranhão. Email: anaflaviadahora@hotmail.com
} 
Pelham - version IV). In the process of data, the software SPSS (Statistical Package for Social Sciense), version 22.0 was used. The results indicate that there are symptoms of inattention in autistic children and adolescents according to the parents' perception. The research contributed to the expansion of the studies concerning the subject of ADHD, as well as to point out possible correlations between ADHD and ASD. Studies on the prevalence of ADHD as a comorbid disorder of ASD, as well as the extension of the clinical sample, is suggested for future research.

Keywords: Autism; Parents; CBCL.

\section{INTRODUÇÃO}

Questionamentos relacionados aos transtornos do neurodesenvolvimento têm se expandido ao longo do tempo, visto que estes têm acarretado uma série de dúvidas a respeito de suas características. A exemplo disso, há inúmeras investigações relacionadas à temática Transtorno do Déficit de Atenção e Hiperatividade (TDAH). Alicerçado nisso, o referente estudo busca acentuar as investigações relativas aos sintomas do TDAH em crianças e adolescentes com diagnóstico do Transtorno do Espectro Autista (TEA), assim como identificar a percepção dos pais no que concerne a tais sintomas.

No que tange à definição do TDAH, o Manual Estatístico dos Transtornos Mentais-DSM-5 (APA, 2014) classifica-o como um transtorno do neurodesenvolvimento, cujos sintomas característicos são: desatenção, hiperatividade e impulsividade. Este habitualmente permanece até a vida adulta, de modo a acarretar danos significativos no âmbito profissional, acadêmico e social do indivíduo.

O transtorno tem acarretado uma série de preocupações no que se refere à sua etiologia, considerada multifatorial, envolvendo fatores genéticos e ambientais, assim como em relação às consequências desencadeadas pelo transtorno. Os sintomas do TDAH manifestam-se de maneira precoce, cujo diagnóstico pode ser realizado logo no início da infância, pois seus sintomas são facilmente observados por pais, cuidadores e professores, tendo em vista que estes acompanham a criança por um longo período de tempo, de modo a identificar possíveis dificuldades relacionadas à realização de tarefas que exigem atenção, cautela e concentração.

De acordo com Missawa e Rossetti (2014), o TDAH é constituído por três características, sendo elas: a impulsividade, a hiperatividade e a dificuldade de atenção. Em decorrência do grande aumento de diagnósticos do transtorno, este apresenta-se 
como algo emergente no contexto atual, evidenciando a importância do campo da psicologia estender seus estudos relacionados ao TDAH.

Tendo em vista a importância de se investigar a percepção dos pais a respeito do processo de desenvolvimento de seus filhos, no que tange à sua contribuição para a obtenção de informações da história de vida do indivíduo e realização de possíveis diagnósticos, a referente pesquisa objetivou apontar a percepção dos pais relativa aos sintomas do TDAH, como desatenção, hiperatividade e impulsividade em crianças e adolescentes com idade de 6 a 12 anos, que possuem diagnóstico clínico de TEA.

\section{MATERIAL E MÉTODOS}

O estudo é do tipo preliminar, de cunho exploratório e caráter quantitativo. Esta apresenta resultados preliminares de uma amostra clínica sobre os sintomas do TDAH apresentados por crianças com o diagnóstico de TEA, sob a ótica de seus pais que buscaram serviços de atendimento psicológico aos filhos fornecido pelo Laboratório de Avaliação, Pesquisa e Intervenção em Transtorno do Espectro Autista (LAPITEA), onde se utiliza o método de Análise do Comportamento Aplicada (ABA), estando sob acompanhamento há 4 meses.

A pesquisa foi realizada no Laboratório de Avaliação, Pesquisa e Intervenção em Transtorno do Espectro Autista (LAPITEA), vinculado à Universidade CEUMA, situado na cidade de São Luís - MA, Brasil. Sua aplicação ocorreu entre os meses de setembro a outubro de 2017. Participaram do estudo preliminar 8 pais de crianças e adolescentes com idade entre 6 a 12 anos, com diagnóstico clínico de autismo. Os participantes integram-se a uma amostra maior cujos filhos são acompanhados pelo LAPITEA. A amostra é composta por pais que preencheram devidamente a escala CBCL/6-18 (Child Behaviour Checklist) e o questionário SNAP-IV (Swanson, Nolan e Pelham - vesão IV). Utilizou-se como critério para a composição da amostra ter filhos com idade entre 6 a 12 anos, pois a escala utilizada é específica para essa faixa etária.

Inicialmente, foi realizada a triagem com os pais para identificar os que cumpriam o critério de inclusão do estudo. Posteriormente, foram repassadas todas as informações relacionadas ao processo de coleta de dados, para então ocorrer à efetivação da pesquisa. Logo após foram entregues a escala e questionário aos pais, para que estes respondessem. Para a realização da análise de dados tanto do CBCL, quanto do SNAP-IV, utilizou-se o software SPSS (Statistical Packager for Social Science), 
versão 22.0, que forneceu uma análise estatística dos resultados obtidos através das respostas dos integrantes da pesquisa. O presente estudo constitui-se como projeto guarda-chuva, estando vinculado ao projeto do LAPITEA, sendo este aprovado pelo Comitê de Ética em Pesquisa (CEP) da Universidade CEUMA, com o registro de CAAE: 70185617.1.0000.5084.

\section{RESULTADOS}

O ponto de corte utilizado para o instrumento de coleta de dados CBCL foi $(\mathrm{T}=60)$ para os problemas referentes à internalização e externalização identificados nas escalas da tabela 05. No que se refere ao escore $\mathrm{T}$, utilizou-se como critério de classificação de grupo normal (não clínico) o ponto de corte de no máximo 60 pontos, para o grupo limítrofe de 60 a 64 pontos e o grupo clínico superior a 64 . No estudo em questão, foram classificados em casos clínicos e não clínicos. Achenbach (1991) recomenda, no intuito de haver redução de dados, que se incluam no grupo clínico aquelas crianças que receberam classificação de grupo limítrofe.

$\mathrm{Na}$ amostra investigada, utilizando-se o instrumento CBCL (tabela 01), identificou-se a presença de sintoma de desatenção, dos quais se apresentam com maior grau de intensidade os seguintes comportamentos: "presença de desatenção e se distrai facilmente na realização de atividades" $(\mathrm{M}=1,38 ; \mathrm{DP}=916)$; "Comportamento de maneira infantil" $(\mathrm{M}=1,38 ; \mathrm{DP}=744)$; "Dificuldade de manter a atenção por tempo prolongado" ( $M=1,50 ; \mathrm{DP}=756)$; "Frequentemente o olhar fica estagnado como se estivesse olhando para o vazio" $(\mathrm{M}=1 ; \mathrm{DP}=756)$.

Tabela 01. Lista de sintomas do CBCL.

\begin{tabular}{|l|r|r|r|}
\hline CBCL- Escala Problemas de Atenção & N & Média (M) & Desvio Padrão (DP) \\
\hline Comporta-se de modo infantil & 8 & 1,38 &, 744 \\
Não consegue terminar as coisas que começa & 8 &, 88 &, 641 \\
Não se concentra, não consegue prestar atenção por muito tempo & 8 & 1,50 &, 756 \\
É agitado, não para quieto & 8 &, 75 &, 707 \\
Parece estar confuso, atordoado & 8 &, 25 &, 463 \\
Fica no "mundo da lua", perdido nos pensamentos & 8 &, 88 &, 835 \\
É impulsivo, age sem pensar & 8 &, 75 &, 707 \\
Não vai bem na escola & 8 &, 13 &, 354 \\
É desatento, distrai-se com facilidade & 8 & 1,38 &, 916 \\
Fica com o olhar parado, "olhando o vazio" & 8 & 1,00 &, 756 \\
& & & \\
\hline
\end{tabular}

Fonte: CBCL/6-18. 
Com a utilização do instrumento SNAP-IV (tabela 02), identificou-se a existência de sintomas de desatenção dentre os quais destacam-se: "Frequentemente, evita se envolver em atividades que requerem um considerável nível de esforço mental" ( $\mathrm{M=2,63;} \mathrm{DP}=518)$; "Não consegue terminar tarefas escolares e deveres que lhe são atribuídos, apresentando dificuldade de seguir instruções até o final" $(\mathrm{M}=2,50 ; \mathrm{DP}=756)$; "Apresenta dificuldade no que se refere à organização de suas tarefas e atividades que são desenvolvidas diariamente" $(M=2,50$; $\mathrm{DP}=756)$; "Manifesta dificuldade no desempenho de atividades relacionadas ao lazer" $(\mathrm{M}=2,13$; $\mathrm{DP}=641$ ); “ Por diversas vezes, comete erros no desempenho de atividades escolares em decorrência do descuido e dificuldade de prestar atenção durante a execução de tais atividades" $(\mathrm{M}=2,13 ; \mathrm{DP}=641)$.

Tabela 02. Lista de sintomas do SNAP-IV.

\begin{tabular}{|c|c|c|c|}
\hline SNAP-IV & $\mathrm{N}$ & $\begin{array}{l}\text { Média } \\
\text { (M) }\end{array}$ & $\begin{array}{l}\text { Desvio Padrão } \\
\text { (DP) }\end{array}$ \\
\hline $\begin{array}{l}\begin{array}{l}\text { Não consegue prestar muita atenção a detalhes ou comete erros por } \\
\text { descuido nos trabalhos da escola ou tarefas }\end{array} \\
\end{array}$ & 8 & 2,13 & 641 \\
\hline Tem dificuldade de manter a atenção em tarefas ou atividades de lazer & 8 & 2,13 & ,641 \\
\hline Parece não estar ouvindo quando se fala diretamente com ele & 8 & 1,25 & ,463 \\
\hline $\begin{array}{l}\text { Não segue instruções até o fim e não termina deveres de escola, tarefas } \\
\text { ou obrigações. }\end{array}$ & 8 & 2,50 & 756 \\
\hline Tem dificuldade para organizar tarefas e atividades & 8 & 2,50 &, 756 \\
\hline $\begin{array}{l}\text { Evita, não gosta ou se envolve contra a vontade em tarefas que exigem } \\
\text { esforço mental prolongado }\end{array}$ & 8 & 2,63 & ,518 \\
\hline Perde coisas necessárias para atividades & 8 & ,88 & ,835 \\
\hline Distrai-se com estímulos externos & 8 & 2,13 & ,641 \\
\hline É esquecido em atividades do dia-a-dia & 8 & 1,50 &, 535 \\
\hline Mexe com as mãos ou os pés ou se remexe na cadeira & 8 & 2,13 & 641 \\
\hline $\begin{array}{l}\text { Sai do lugar na sala de aula ou em outras situações em que se espera que } \\
\text { fique sentado }\end{array}$ & 8 & 2,13 & 641 \\
\hline $\begin{array}{l}\text { Corre de um lado para outro ou sobe demais nas coisas em situações em } \\
\text { que isto é inapropriado }\end{array}$ & 8 & 1,63 & 1,188 \\
\hline $\begin{array}{l}\text { Tem dificuldade em brincar ou envolver-se em atividades de lazer de } \\
\text { forma calma }\end{array}$ & 8 & 1,25 & 463 \\
\hline Não para ou frequentemente está a "mil por hora" & 8 & 1,00 &, 000 \\
\hline Fala em excesso & 8 & ,63 & ,744 \\
\hline $\begin{array}{l}\text { Responde às perguntas de forma precipitada antes delas terem sido } \\
\text { terminadas }\end{array}$ & 8 & ,38 & ,518 \\
\hline Tem dificuldade de esperar sua vez & 8 & 1,75 & ,463 \\
\hline Interrompe os outros ou se intromete & 8 & ,38 &, 518 \\
\hline
\end{tabular}

Fonte: DSM-5 (APA, 2014, p. 59-60).

Para a classificação do ponto de corte clínico e não clínico da tabela 04 que se refere ao instrumento SNAP-IV, procedeu-se do subsequente modo: se de 1 a 9 existir, no mínimo, 6 itens respondidos como "BASTANTE" ou "DEMAIS", considera-se que, para uma criança ou adolescente, há sintomas de desatenção além do esperado; se de 10 a 18 existir, no mínimo, 6 itens respondidos como "BASTANTE" ou "DEMAIS", 
considera-se que, para uma criança ou adolescente, tem-se sintomas de hiperatividade e impulsividade além do esperado.

Tabela 03. Classificação do SNAP-IV.

\begin{tabular}{lllll}
\hline \multirow{2}{*}{ SNAP-IV } & \multicolumn{2}{c}{$\begin{array}{c}\text { Clínico } \\
\end{array}$} & \multicolumn{2}{c}{$\begin{array}{c}\text { Não Clínico } \\
(<\mathbf{1 2})\end{array}$} \\
\cline { 2 - 5 } & $\mathrm{N}$ & $\%$ & $\mathrm{~N}$ & $\%$ \\
\hline Déficit de Atenção & 7 & 87,5 & 1 & 12,5 \\
\hline Hiperatividade/Impulsividade & 0 & 0 & 8 & 100 \\
\hline
\end{tabular}

Dentro da amostra investigada, utilizando o instrumento SNAP-IV, 87,5\% dos sujeitos apresentaram resultados significativos de déficit de atenção, segundo a percepção dos pais, classificando-se como grupo clínico e $12,5 \%$ dos sujeitos como não clínico. Já em relação aos sintomas de hiperatividade e impulsividade, não foram encontrados resultados significativos (tabela 03).

Tabela 04. Classificação do CBCL.

\begin{tabular}{|c|c|c|c|c|}
\hline \multirow[t]{2}{*}{ CBCL } & \multicolumn{2}{|c|}{$\begin{array}{l}\text { Clínico } \\
(\geq 64)\end{array}$} & \multicolumn{2}{|c|}{$\begin{array}{l}\text { Não Clínico } \\
(<63)\end{array}$} \\
\hline & $\mathrm{N}$ & $\%$ & $\mathrm{~N}$ & $\%$ \\
\hline Problemas de Atenção & 6 & 75,0 & 2 & 25,0 \\
\hline Violação de Regras & 1 & 12,5 & 7 & 87,5 \\
\hline Comportamentos Agressivos & 0 & 0 & 8 & 100 \\
\hline
\end{tabular}

No resultado da aplicação da escala CBCL, 75,0\% dos sujeitos apresentaram resultados significativos de problemas de atenção, classificando-se como grupo clínico e $25,0 \%$ dos indivíduos como não clínico, 12,5\% apresentaram resultados de violação de regras, classificando-se como grupo clínico e $87,5 \%$ como não clínico. No que tange aos comportamentos agressivos, nenhum dos sujeitos apresentou tal resultado (tabela 04).

Referente aos dados sociodemográficos da amostra clínica (tabela 05), os achados mais significativos referem-se à situação laboral, cuja porcentagem de pais que ambos trabalham é de 50\%, no que tange à escolaridade, 50\% deles possuem ensino superior e quanto à frequência das crianças na escola, 62\% deles frequentam escola convencional, $37,5 \%$ não frequentam a escola e nenhum deles frequenta a escola especial.

Tabela 05. Dados sociodemográficos. 


\begin{tabular}{|lcc|}
\hline Dados sociodemográficos & N & $\%$ \\
Situação Laboral & & \\
O pai é o único provedor & 3 & $37,5 \%$ \\
A mãe é a provedora & 1 & $12,5 \%$ \\
Ambos trabalham & 4 & $50 \%$ \\
Escolaridade dos pais & & \\
Ensino Médio & 3 & $37,5 \%$ \\
Ensino Superior & 4 & $50 \%$ \\
Ensino médio incompleto & 1 & $12,5 \%$ \\
Frequência das crianças na escola & & \\
Frequenta a escola convencional & 5 & $62,5 \%$ \\
Frequenta escola especial & 0 & \\
Não frequenta a escola & 3 & $37,5 \%$ \\
\hline
\end{tabular}

\section{DISCUSSÃO}

Os resultados apontados no presente estudo, caracterizado como estudo preliminar, indicam a presença de sintomas de desatenção na amostra clínica, segundo a ótica dos pais, identificados tanto no instrumento de coleta de dados CBCL, como no SNAP-IV, apontando para a existência de uma concordância de resultados entre os dois instrumentos utilizados para coleta de dados. De acordo com Souza et al. (2007), o TDAH pode apresentar-se como transtorno comórbido do TEA, afirmando que os sintomas de impulsividade, hiperatividade e desatenção muitas vezes apresentados por sujeitos com autismo assemelham-se a aqueles existentes em indivíduos com TDAH, sintomas estes dos quais professores e pais queixam-se frequentemente, de modo a não descartar a possibilidade de um diagnóstico de TDAH em indivíduos com autismo.

No que tange aos sintomas do TDAH apontados pelo DSM-5 (APA, 2014), alguns deles são: não consegue se concentrar; distrai-se facilmente; diante de atividades que requer esforço mental, frequentemente, se esquiva; não termina funções que lhe são designadas, como atividades do trabalho ou da escola. Os achados da presente pesquisa apontam para a existência de tais sintomas na amostra clínica, os quais apresentaram-se de modo significativo, sinalizando uma possível existência do TDAH como comorbidade do autismo.

$\mathrm{Na}$ amostra em questão, observou-se a presença de um alto grau de dificuldade de envolver-se em tarefas que necessitam da presença duradoura de esforço mental, tendo o resultado de $(\mathrm{M}=2,63 ; \mathrm{DP}=518)$, o que comprova a existência de problemas de atenção, visto que para a realização de atividades diárias, faz-se necessária a existência de um esforço mental, exigindo atividade de concentração. De acordo com Rohde et al. (2000), a predominância dos sintomas característicos de desatenção acarreta um maior nível de comprometimento acadêmico, resultando numa série de prejuízos para a vida 
do sujeito, pois este apresenta dificuldade em manter a atenção, atividade esta caracterizada como de extrema importância para o processo de aprendizagem, apresentando-se com maior frequência em pessoas do sexo feminino.

$\mathrm{Na}$ análise referente aos grupos clínicos e não clínicos dos dois instrumentos utilizados, ambos apontaram para a presença de problemas de atenção, com resultado de 87,5\% do grupo clínico no SNAP-IV e 75,0\% no CBCL, sinalizando concordância entre os resultados de ambos os instrumentos, o que torna os achados da pesquisa mais significativo e fidedigno, visto que apontam para um resultado em comum.

No que se refere às características sociodemográficas da amostra no que tange à situação laboral, observou-se o predomínio de que tanto o pai quanto a mãe trabalham. No que se refere ao nível de escolaridade dos pais, metade possui nível superior completo, os demais possuem ensino médio completo e incompleto. A maioria das crianças e adolescentes frequenta a escola, porém apresentam dificuldades no desenvolvimento das atividades atribuídas a eles em tal âmbito, dificuldades estas recorrentes principalmente pelo comprometimento da capacidade de sustentar a atenção.

Um estudo realizado por Pondé, Novais e Losapio (2010), com 32 crianças de uma escola localizada na cidade de Salvador - BA, especializada em crianças autistas que visava identificar a existência de sintomas do TDAH em tais sujeitos, utilizou como instrumento de pesquisa a entrevista K-SDAS PL na versão brasileira, apontou como resultado a presença dos sintomas do TDAH em 53,1\% dos indivíduos, sendo que destes 5,8\% apresentaram sintomas característicos de desatenção.

Esses achados corroboram com os resultados da presente pesquisa ao apontar a existência de uma predominância dos sintomas de desatenção manifestados pela amostra clínica, se visto que existe a possibilidade do TDAH apresentar-se como comorbidade do TEA, de modo a sinalizar a presença significativa de sintomas do TDAH em sujeitos com diagnóstico clínico de autismo. Outro estudo realizado nos Estados Unidos por Leyfer et al. (2006), tendo como instrumento de coleta de dados o Autism Comorbidity Interview-Present and Lifetime version (ACI-PL), cuja entrevista foi aplicada com os pais de 109 crianças autistas com faixa etária de 5 a 17 anos, tendo como objetivo diagnosticar possíveis transtornos psiquiátricos em sujeitos que possuem diagnóstico clínico de autismo, apontou como resultado que $55 \%$ das crianças inclusas na pesquisa possuíam TDAH, relacionado aos critérios diagnósticos, outras $24 \%$ por pouco não o atingiram. 
No que se refere à presença de comorbidade em indivíduos com diagnóstico de TEA, o DSM-5 (APA, 2014) destaca que, aproximadamente, 70\% deles podem apresentar algum tipo de transtorno caracterizado como comórbido, enfatizando que, ao serem preenchidos os critérios para o TDAH e para o TEA, faz-se necessária a efetivação dos diagnósticos para ambos os transtornos.

É válido ressaltar que ocorreu uma mudança do DSM-4 para o DSM-5, em relação às comorbidades do TEA, na sua quarta versão, este descartava a possibilidade da realização de um diagnóstico de TDAH em indivíduos autistas. Já na versão atual, este afirma que isso é possível, informação esta que está em concordância com os resultados da referente pesquisa, ao indicar a presença de sintomas de desatenção em crianças e adolescentes com diagnóstico de TEA.

Os resultados da presente pesquisa sinalizam para a necessidade de um olhar mais atento para as especificidades do TDAH, visto que esse transtorno tem afetado de maneira significativa a vida dos sujeitos que o possuem. Igualmente, requer uma prática profissional que leve em consideração a importância do depoimento dos pais para a realização de um possível diagnóstico, já que estes apresentam-se como fontes relevantes de informação a respeito do desenvolvimento dos filhos.

\section{CONCLUSÕES}

Diante da investigação das concepções dos pais acerca dos sintomas do TDAH em filhos autistas, percebe-se que estes possuem a capacidade de identificação precisa de tais sintomas e como estes se manifestam em diversos contextos, de maneira a formular um relato conciso sobre os sintomas, embora muitos não tenham o conhecimento do que de fato caracteriza o TDAH.

Aponta-se como uma das principais contribuições desse estudo para a produção científica a ampliação de estudos sobre a temática TDAH, visto que tem crescido o número de diagnósticos de tal transtorno. Em contrapartida, encontra-se pouca literatura no que se refere à percepção dos pais concernente à presença dos sintomas do TDAH em crianças e adolescentes com TEA. Cabe aqui apontar para a originalidade desta pesquisa, visto que não se encontrou na literatura estudos com o mesmo objetivo.

A importância desse estudo para a psicologia refere-se principalmente ao caráter de relevância desta, uma vez que cabe ao psicólogo enquanto profissional preocupado com o bem-estar dos sujeitos estar atento para as especificidades do 
transtorno, assim como ter conhecimento das concepções dos pais a respeito do desenvolvimento dos filhos, tendo em vista a grande importância que eles exercem para o processo de investigação dos sintomas característicos do TDAH.

Todavia, torna-se necessário apontar algumas limitações encontradas, tais como o tamanho da amostra considerada pequena, a falta de disponibilidade dos pais para responderem aos instrumentos utilizados na pesquisa, a falta de um grupo controle e, por fim, mas não menos relevante, a inexistência de pesquisas que objetivavam apontar a percepção dos pais a respeito da manifestação dos sintomas do TDAH em autistas. Sugere-se, para pesquisas futuras, a ampliação da amostra clínica, assim como o estudo da prevalência do TDAH como transtorno comórbido do TEA.

\section{REFERÊNCIAS}

ACHENBACH, Thomas M. Integrative guide for the 1991 CBCL/4-18, YSR and TRF profiles. 1. ed. Vermont: Burlington, 1991.

AMERICAN PSYCHIATRIC ASSOCIATION. DSM-5: manual diagnóstico e estatístico de transtornos mentais. 5 ed. Porto Alegre: Artmed, 2014.

LEYFER, Ovsanna T. et al. Comorbidades Psiquiátricas em Crianças com Autismo: Desenvolvimento de Entrevista e Taxa de Transtornos, Journal of Autism and Developmental Disorders, v. 36, n. 7, 2006. Disponível em: <http://www.ama.org.br/site/images/home/Artigos/DesenvolvimentodeEntrevista.pdf >. Acesso em: 13 out. 2017.

MISSAWA, Daniela D. A.; ROSSETTI, Claudia B. Psicólogos e TDAH: possíveis caminhos para diagnóstico e tratamento. Construção Psicopedagógica, São Paulo, v. 22, n. 23, p. 81-90, 2014. Disponível em:

<http://pepsic.bvsalud.org/pdf/cp/v22n23/07.pdf〉. Acesso em: 25 set. 2017.

PONDÉ, Milena P.; NOVAES, Camila M.; LOSAPIO, Mirella F. Frequência de sintomas de déficit de atenção e hiperatividade em crianças autistas. Arquivos de Neuro-Psiquiatria, São Paulo, v. 68, n. 1, p. 103-106, 2010. Disponível em: <http://www.scielo.br/pdf/anp/v68n1/a22v68n1.pdf>. Acesso em: 15 set. 2017.

ROHDE, Luís A. et al. Transtorno de déficit de atenção/hiperatividade. Revista Brasileira de Psiquiatria, São Paulo, v. 22, n. 2, p. 7-11, 2000. Disponível em: <http://www.scielo.br/pdf/rbp/v22s2/3788.pdf>. Acesso em: 07 set. 2017.

SOUZA, Izabella G. S. et al. Dificuldades no diagnóstico de TDAH em crianças. Jornal Brasileiro de Psiquiatria, Rio de Janeiro, v. 56, n. 1, p. 14-18, 2007. 
Disponível em: <http://www.scielo.br/pdf/jbpsiq/v56s1/a04v56s1.pdf>. Acesso em: 14 out. 2017. 